\title{
Growing with the world: rapid development of the zebrafish research in China and the China Zebrafish Resource Center
}

\author{
XIE XunWei, PAN LuYuan \& SUN YongHua* \\ China Zebrafish Resource Center, State Key Laboratory of Freshwater Ecology and Biotechnology, Institute of Hydrobiology, Chinese \\ Academy of Sciences, Wuhan 430072, China
}

Received August 4, 2014; accepted November 21, 2014

Citation: Xie XW, Pan LY, Sun YH. Growing with the world: rapid development of the zebrafish research in China and the China Zebrafish Resource Center. Sci China Life Sci, 2015, 58: 396-399, doi: 10.1007/s11427-015-4816-5

Zebrafish (Danio rerio), a small tropical freshwater fish native to east India, has rapidly emerged as a popular vertebrate model organism in the last two decades. There are multiple advantages of using zebrafish in biological research, including its small size, short generation time of 2-3 months, external fertilization and development, high fecundity without seasonal variation, transparent embryos, and inexpensive maintenance. The well-annotated zebrafish genome contains more than 26,000 protein-coding genes, sharing over $80 \%$ similarity with human genome, and approximately $70 \%$ of human genes have its zebrafish orthologues. Drug administration in zebrafish can be as simple as adding chemicals into fish water, and therefore it is doable to design projects with a small budget compared to mice and rats. Zebrafish is also famous for its amazing regenerative abilities, for instance, it can regenerate fins, hair cells, retina, even heart and brain. All these features make zebrafish a valuable animal model to dissect the mechanisms of biological processes and human diseases [1,2].

Zebrafish research started at University of Oregon in 1970s, when Dr. George Streisinger and his colleagues produced the first batch of zebrafish homozygous clones by early hydrostatic pressure method. In 1990s, the well-known Boston and Tübingen screens have successfully identified several thousand mutations with embryonic phenotypes that consolidated the importance of zebrafish as an animal model in scientific research [3]. Since then the

*Corresponding author (email: yhsun@ihb.ac.cn) zebrafish model has been successful in forward genetic studies, which use alkylating or insertional mutagens to induce mutagenesis following with the screening of observable phenotypes.

The Sanger Institute started the zebrafish genome sequencing project in 2001. Several assemblies have been generated and further improvements are released continuously. Accompanying with the full sequencing of the zebrafish genome, reverse genetic approaches have played more and more important roles in obtaining loss-of-function mutants and precise genome editing. Especially in recent years, the engineered endonucleases, e.g., transcription activator-like effector nuclease (TALEN) and guide RNACRISPR associated 9 nuclease (CRISPR/Cas9) [4], can efficiently create double-strand breaks (DSBs) in target genes, and subsequently induce random indel into the DSB sites, or facilitate the introduction of exogenous sequences. In addition, abundant transgenic fish resources have been generated by Tol 2 transposon system. For instance, ubiquitous or targeted expression of fluorescent proteins, and the use of GAL4/UAS system and CRE-loxP system in the zebrafish genome provide powerful tools to observe embryonic development and analyze gene functions [5].

While first established as a model to study vertebrate embryonic development, now zebrafish has been widely applied in the studies of genetics, organogenesis, regeneration, neurobiology, modelling human diseases, drug screening and optimization, environmental biology and so on. In a recent assessment of PubMed publications, it is shown that 
zebrafish publications display the sharpest increase compared with other animal models in the last 10 years [6].

In China, zebrafish was firstly adopted in lab research in early 1990s, and since 2000 the zebrafish research community has been rapidly growing. Based on the search results in Web of Science ${ }^{\mathrm{TM}}$ Core Collection (v5.16), in 2001, the proportion of zebrafish-related papers published by Chinese investigators took up only $1.8 \%$ in the total zebrafish papers published worldwide. This proportion has shown a sharp increase since then with the increase of total zebrafish publications worldwide. It first surpassed $10 \%$ in 2010, and continuously increased to $16.8 \%$ in 2014 , runner-up only to the United States (Figure 1A).

Not only is the amount of the zebrafish-related research in China increasing, but also the breadth of areas. Based on the same database, zebrafish-related work focuses mainly on developmental biology, biochemistry and molecular biology, cell biology, genetics, neuroscience, zoology, toxicology, environmental sciences, freshwater biology, etc. From 2000 to 2014, papers published in the above top 10 research areas took up $79 \%(20,939 / 26,490)$ of all zebrafish-related publications over the world. In China and in the same period, $73 \%(1,922 / 2,615)$ papers were published in the top 10 research areas, and all aspects were involved, which shows a wide range of research interests in China (Figure 1B). Notably, the largest proportions here are in the fields of environmental sciences and toxicology, suggesting that zebrafish has been widely utilized to resolve the current environmental issues in China.

In recent years, more and more high-quality papers have

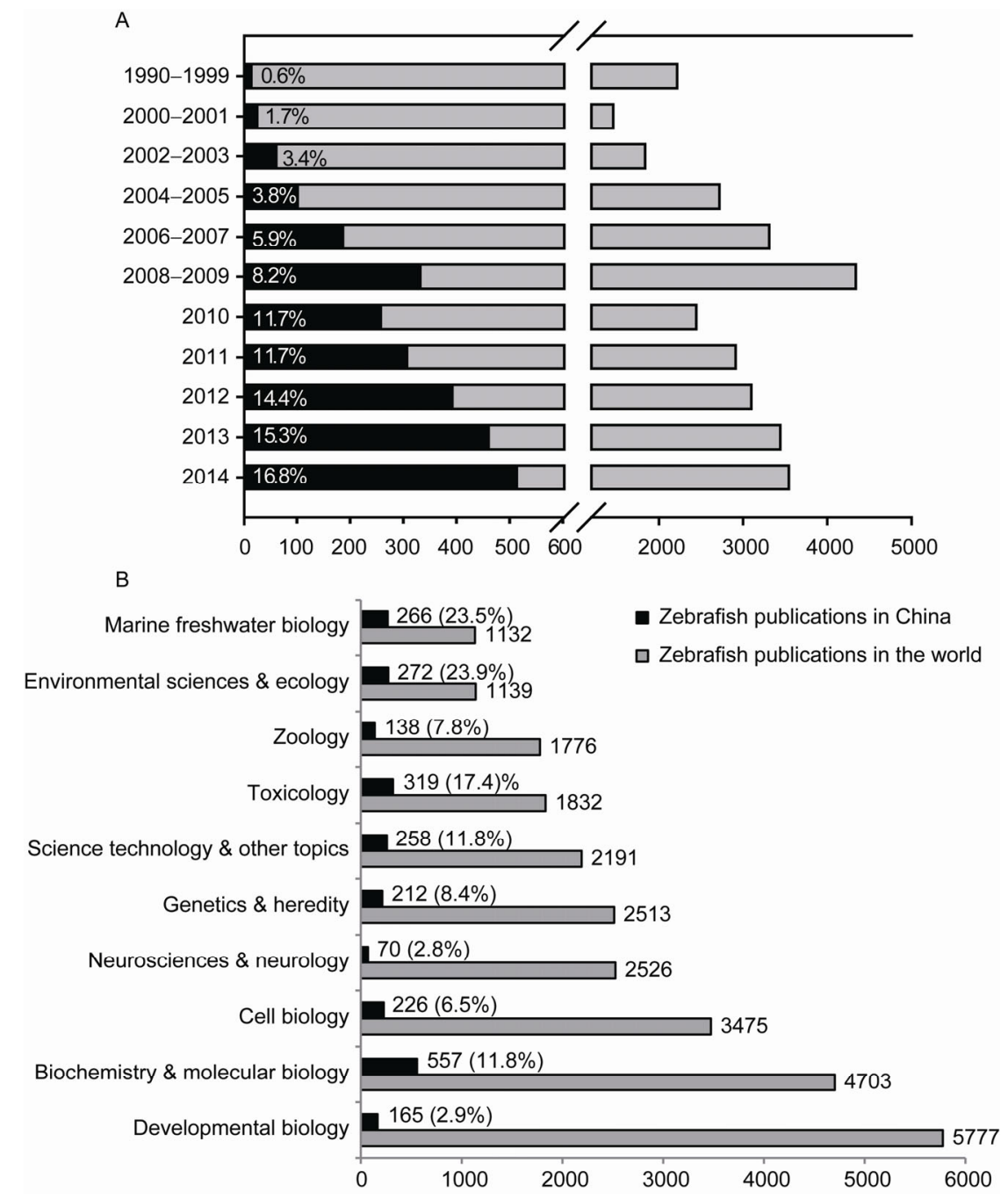

Figure 1 A historical perspective of zebrafish research in China. All data was acquired from Web of Science ${ }^{\mathrm{TM}}$ Core Collection (v5.16). Grey bars, the zebrafish-related publications around the world (topic: zebrafish); black bars, the zebrafish-related publication in China (topic: zebrafish; address: China). A, The proportion of zebrafish-related papers published by Chinese investigators in those published worldwide from 1990 to 2014 . $X$ axis is the amount of publications and $Y$ axis is time. The percentage numbers in each bar represent the proportion of publications in China in corresponding year(s). The breaks on $x$ axis indicate the amount from 600 to 1,200. B. The amount of zebrafish publications in the top 10 research areas in China and worldwide. $X$ axis is the amount of publications and $Y$ axis lists the research fields. Number on the top of each bar indicates the amount of publications in each research area. Numbers in parentheses indicate the proportions of the publications in China in each research area. 
been published by Chinese researchers in the major journals, such as Cell, Science, and Nature. In several representative zebrafish-related fields, such as embryonic development and genetic methodology, Chinese zebrafish research community had quite a lot internationally recognized achievements. For instance, as the first zebrafish lab in China, Meng's team at Tsinghua University and collaborators have studied the roles of Nodal, BMP, FGF and Wnt/beta-catenin signaling pathways in zebrafish embryogenesis, and identified a series of pivotal genes mediating mesoderm induction and dorsal-ventral patterning [3]. In the genetic methodology area, Chinese fish geneticists made significant progress in developing transgenic fish and heritable knock-out technologies using TALENs and CRISPR/Cas9, which largely enriched the zebrafish reverse-genetic toolbox [7].

In 2010, when the proportion of zebrafish-related papers from China surpassed 10\%, the First China Zebrafish Research Conference was held at Zhejiang University with about 50 participants, and this number doubled in 2011 and exceeded 400 in the third conference in 2013. The First Chinese Zebrafish Principle Investigator (PI) Meeting was held in Wuhan in 2012 with over 70 PIs attending, and this number also doubled in the second meeting in 2014. The Chinese zebrafish community decided that both meetings will be held every other year-national conference is in the odd-numbered and PI meeting in the even-numbered years. According to a recent survey, there are over 300 labs in China that utilize zebrafish in their work. With the fast rising of zebrafish-related research, the demands of zebrafish resource are also increasing continuously in China.

Resource centers are always the sine qua non of research development. The Zebrafish International Resource Center (ZIRC), housed in the University of Oregon campus, is the first and largest zebrafish resource institution in the world. ZIRC provides the resources of fish lines, materials and information to whole zebrafish community around the world. However, because of the difficulties of importing and exporting living fish and international shipping, several similar zebrafish resource centers were established in other regions for local requirements.

To meet the growing needs of zebrafish strains and technical support, the China Zebrafish Resource Center (CZRC) was established in October, 2012 in Wuhan at the Institute of Hydrobiology (IHB), Chinese Academy of Sciences (CAS), a key research center of freshwater fish biology. CZRC is a non-profit organization jointly supported by the Ministry of Science and Technology of China, and the CAS.

IHB provided and furnished a whole floor of $580-\mathrm{m}^{2}$ area, in which CZRC has built two main fish rooms, a quarantine fish room, a nursery room, a cryopreservation lab, a molecular biology lab, a cell culture room and a microscope room. Two main fish rooms cover an area of $250 \mathrm{~m}^{2}$ with the capacity of more than 10,000 fish tanks. CZRC is also equipped with two ultra-large liquid nitrogen storage tanks which can hold over 40,000 tubes for zebrafish sperm cryo- preservation.

The main missions of CZRC include collecting and preserving the existing zebrafish resources, developing new fish lines and research tools, distributing the resources as request, and providing technical and informatics support. After two years' development, till late 2014, CZRC has maintained more than 400 mutant and transgenic zebrafish lines, and a number of DNA plasmids and cell lines. In addition to providing the regular fish resources, CZRC also provides zebrafish-related technical assistances, involving technical training, such as fish husbandry and regular approaches of molecular biology, and gene knock-out or transgenic services. CZRC now hosts the biennaleese Zebrafish PI Meeting, and yearly training workshop of zebrafish technology. CZRC is also planning to build an open database for not only local resource management, but also cloud service to Chinese zebrafish labs.

In 2013, 35 labs in China started the Zebrafish All Gene Knock-Out Consortium for Chromosome 1. Now this consortium has more than 40 members, and the joint project, the Zebrafish All Gene Knock-out Project for Chromosome 1 , is ongoing. The project plans to knock out more than 1,000 genes on zebrafish chromosome 1 using CRISPR/ Cas9 system. CZRC is the project coordinator, and all mutant lines built in the project will be tested and preserved in CZRC and published as open resources to the whole research field. With the project completing, CZRC will expand its fish stocks to 2,000 lines at the end of 2015, and become the largest zebrafish resource center in Asia.

Zebrafish, a young and promising vertebrate system, contributes more and more to our understanding of biological processes and human diseases. Going along with the fast growing of zebrafish research in the world, the Chinese zebrafish community will accomplish more significant achievements in solving a series of advanced scientific issues, and CZRC will undoubtedly play a pivotal role in supporting zebrafish-related studies in China.

1 Patton EE, Zon LI. The art and design of genetic screens: zebrafish. Nat Rev Genet, 2001, 2: 956-966

2 Howe K, Clark MD, Torroja CF, Torrance J, Berthelot C, Muffato M, Collins JE, Humphray S, McLaren K, Matthews L, McLaren S, Sealy I, Caccamo M, Churcher C, Scott C, Barrett JC, Koch R, Rauch GJ, White S, Chow W, Kilian B, Quintais LT, Guerra-Assunção JA, Zhou Y, Gu Y, Yen J, Vogel JH, Eyre T, Redmond S, Banerjee R, Chi J, Fu B, Langley E, Maguire SF, Laird GK, Lloyd D, Kenyon E, Donaldson S, Sehra H, Almeida-King J, Loveland J, Trevanion S, Jones M, Quail M, Willey D, Hunt A, Burton J, Sims S, McLay K, Plumb B, Davis J, Clee C, Oliver K, Clark R, Riddle C, Elliot D, Threadgold G, Harden G, Ware D, Begum S, Mortimore B, Kerry G, Heath P, Phillimore B, Tracey A, Corby N, Dunn M, Johnson C, Wood J, Clark S, Pelan S, Griffiths G, Smith M, Glithero R, Howden P, Barker N, Lloyd C, Stevens C, Harley J, Holt K, Panagiotidis G, Lovell J, Beasley H, Henderson C, Gordon D, Auger K, Wright D, Collins J, Raisen C, Dyer L, Leung K, Robertson L, Ambridge K, Leongamornlert D, McGuire S, Gilderthorp R, Griffiths C, Manthravadi D, Nichol S, Barker G, Whitehead S, Kay M, Brown J, Murnane C, Gray E, Humphries M, Sycamore N, Barker D, Saunders D, Wallis J, Babbage A, 
Hammond S, Mashreghi-Mohammadi M, Barr L, Martin S, Wray P, Ellington A, Matthews N, Ellwood M, Woodmansey R, Clark G, Cooper J, Tromans A, Grafham D, Skuce C, Pandian R, Andrews R, Harrison E, Kimberley A, Garnett J, Fosker N, Hall R, Garner P, Kelly D, Bird C, Palmer S, Gehring I, Berger A, Dooley CM, Ersan-Ürün Z, Eser C, Geiger H, Geisler M, Karotki L, Kirn A, Konantz J, Konantz M, Oberländer M, Rudolph-Geiger S, Teucke M, Lanz C, Raddatz G, Osoegawa K, Zhu B, Rapp A, Widaa S, Langford C, Yang F, Schuster SC, Carter NP, Harrow J, Ning Z, Herrero J, Searle SM, Enright A, Geisler R, Plasterk RH, Lee C, Westerfield M, de Jong PJ, Zon LI, Postlethwait JH, Nüsslein-Volhard C, Hubbard TJ, Roest Crollius H, Rogers J, Stemple DL. The zebrafish reference genome sequence and its relationship to the human genome. Nature, 2013, 496:
498-503

3 Jia SJ, Meng AM. The development of zebrafish research in China. Hereditas (Beijing), 2012, 34: 1082-1088

4 Zhang L, Zhou Q. CRISPR/Cas technology: a revolutionary approach for genome engineering. Sci China Life Sci, 2014, 57: 639-640

5 Lawson ND, Wolfe SA. Forward and reverse genetic approaches for the analysis of vertebrate development in the zebrafish. Dev Cell, 2011, 21: 48-64

6 Stewart AM, Braubach O, Spitsbergen J, Gerlai R, Kalueff AV. Zebrafish models for translational neuroscience research: from tank to bedside. Trends Neurosci, 2014, 37: 264-278

7 Ye D, Zhu Z, Sun Y. Fish genome manipulation and directional breeding. Sci China Life Sci, 2015, 58: 170-177

Open Access This article is distributed under the terms of the Creative Commons Attribution License which permits any use, distribution, and reproduction in any medium, provided the original author(s) and source are credited. 